\title{
Structural Analysis of Piezo1 Ion Channel Reveals the Relationship between Amino Acid Sequence Mutations and Human Diseases
}

\author{
Zikai Zhou \\ International Department, Ningbo Xiaoshi High School, Ningbo, China \\ Email: zhouzikai190716@outlook.com
}

How to cite this paper: Zhou, Z.K. (2019) Structural Analysis of Piezo1 Ion Channel Reveals the Relationship between Amino Acid Sequence Mutations and Human Diseases. Journal of Biosciences and Medicines, 7, 139-155.

https://doi.org/10.4236/jbm.2019.712012

Received: October 23, 2019

Accepted: December 7, 2019

Published: December 10, 2019

Copyright $\odot 2019$ by author(s) and Scientific Research Publishing Inc. This work is licensed under the Creative Commons Attribution International License (CC BY 4.0).

http://creativecommons.org/licenses/by/4.0/

\begin{abstract}
Since first identified in 2010, Piezo proteins have been found to perform as pore-forming mechanosensitive ion channels across a wide range of animals. As a Piezo ortholog primarily expressed in mammalian systems, Piezol has been observed to distribute mainly in nonsensory tissues, regulating osmotic homeostasis, proprioception, and light touch. With previous studies on the putative structure of Piezo1, the gating system and several mechanotransduction mechanisms have been proposed. Besides, mutations of specific amino acid sequences in Piezol have been linked to several human diseases such as dehydrated hereditary xerocytosis (DHS) and congenital lymphatic dysplasia (CLD). However, most of these mutations have not been well characterized. To further elucidate the relations between these mutations and diseases, UCSF Chimera is used as the tool to visualize the structural importance of each of these mutated amino acids. With the aid from UCSF Chimera, this study has recorded and interpreted clashes and contacts originated from each of the mutations. Accordingly, specific mechanisms between mutations and human diseases are proposed, which pave the way for healing.
\end{abstract}

\section{Keywords}

Piezo1, Mutation, Protein Structure Human Disease, UCSF Chimera

\section{Introduction}

Piezo channels, first isolated and identified by scientists in 2010, primarily present in the form of two orthologs: Piezol and Piezo2, both contributing to the function of unique mechanosensitive channels [1]-[6]. In the past nine years, by employing the technique of Cryo-EM and single particle analysis, the struc- 
ture of Piezo1 channels has been successfully derived from frozen biomolecular samples. It is shown that the Piezol channel exhibits a trimeric, propeller-like shape, with three identical blades extending out from the central pore in a rotatory manner [7]-[12]. Each of these blades, deflecting at an angle of 100 degrees perpendicular to the membrane, constitutes several repeating amino acid bundles: THUs or transmembrane helical units, within which are four transmembrane helices per THU [8] [10] [11] [12]. To view these Piezo1 repeats more microscopically, the linkage between the first and the second and between the third and the fourth TMs, counted from the proximal to the distal end, are extracellular, whereas the linkage between the second and the third TMs is intracellular [11] [12] [13]. Those three blades all surround a central domain which is composed of one C-terminal domain (CTD), one "cap" or C-terminal extracellular domain (CED), three inner helices $(\mathrm{IH})$, three outer helices $(\mathrm{OH})$, and three anchors and latches [7]-[12]. With a totality of 2521 amino acids for humans (2547 for mice), the "cap" domain has amino acid sequences ranging from 2218 to 2455 , the anchors 2106 to 2175 , the latches 1403 to 1417 [9] [10] [12]. Extending from proximity to the central axis to the so-called "clasp," a beam, roughly at the bottom of the third Piezo repeat or THU, has features that indicate its mechanogating function [11] [12] [13]. It has been deducted that these intracellular beams, three long density rods of about $90 \AA$ in length, are responsible for a lever-like gating mechanism of the central "beam-CTD-anchor-OH-IH" relaying interface that surrounds and forms the central pore module [12]. That is, the beam delivers the mechanical signals from the blades, or the plasma membrane, to the central pore module region [11] [12] [13]. The central pore vestibule, separated by closely interacting amino acid sequences around the central axis, can be divided into EV (extracellular vestibule), MV (membrane vestibule), and IV (intracellular vestibule), whose separation points embrace a pore radius of less than $2 \AA$ in the neighboring state [14]. As the relationship between the free energy and the opening area is proportional [15], Piezo1 has been tested to operate at a relatively substantial threshold area: $400 \mathrm{~nm}^{2}$ for the mid-plane area and $280 \mathrm{~nm}^{2}$ for the projected area, yielding an area of change of $120 \mathrm{~nm}^{2}$ [9]. Therefore, Piezol has a significant requirement for opening and a high cation selectivity [16]. The anchor domains, on the other hand, are of the most considerable evolutionary significance and fixate Piezo1 ion channels inside the membrane [10] [11]. The interaction between Piezo1 and lipid membrane has been demonstrated to cause the curvature on the latter, which suggests a conformational adaptation of cell membrane when Piezo1 channels present [9]. Besides, a model named as "gravitational analog" has been used to explain the mechanism behind Piezol's capability to sense membrane tension [9].

Those structural features and mechanisms all contribute to Piezol's mechanosensitive function. Mechanotransduction, the process in which mechanical signals are converted into electrical impulses, is essential for tension sensing, proprioception, and homeostasis [17] [18] [19]. Identified and interpreted by 
scientists as a mechanosensitive ion channel, Piezol carries out the mechanotransduction process in various animal species, including humans, mice, and zebrafish [8] [9] [10] [11] [19]-[26]. Regarding humans, Piezo1 has been found to play a role in neural stem cell development, osmolarity regulation, and basic sensations [19] [20] [27] [28]. Tissues that have been tested to embrace Piezo1 include erythrocytes, lymphatic valves, and articular cartilage [21] [29]-[34]. To illustrate the importance of Piezo1 in these tissues, mutations in Piezol channels have been observed to lead to multiple human diseases such as dehydrated hereditary xerocytosis (DHS), autosomal recessive congenital lymphatic dysplasia, and pancreatitis [31] [33] [35]-[41]. On top of that, Piezo1 has been identified as an optimizer of human $\mathrm{T}$ cell activation and a prerequisite for bone formation (shown in Figure 1) [24] [42]. Those disease-causing mutations are primarily linked to distortions near the central axis of Piezo1, which indicates alterations in the pore module [43].

Despite the effort taken into researches about Piezo1, there are still aspects remaining unknown: the mechanisms of Piezol functioning inside purified membrane bilayers, the geometry of membrane induced by the indentation and experienced by the channel, the mechanisms for modulators to function, and the effects of functionally distinct mutations on the occurrences of diseases [20]. All these problems need resolution. In this paper, structural analysis of Piezol mutations will be carried out to elucidate the patterns of distorted functions. A total number of 423 cases are considered.

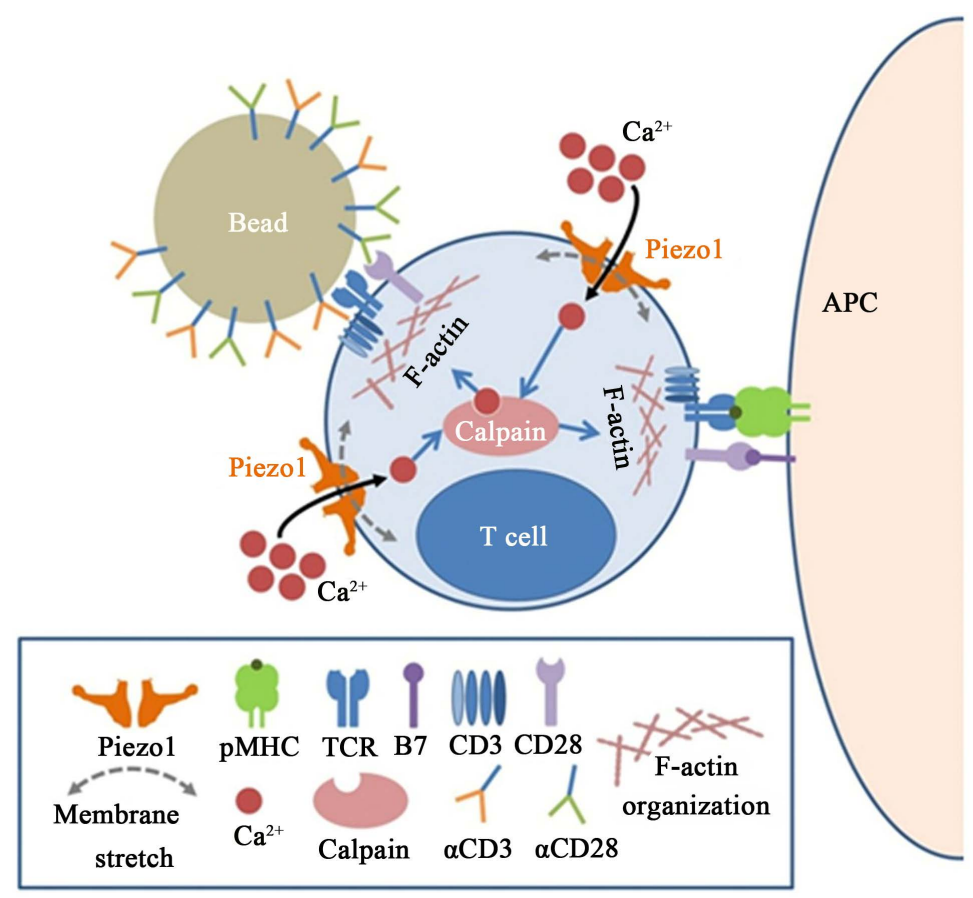

Figure 1. Proposed model for the role of Piezol in human T cell activation. Our data suggest that membrane stretch during immune synapse formation triggers Piezol activation and $\mathrm{Ca}^{2+}$ influx, which, in turn, activate calpain. Calpain activation helps in the organization of the cortical actin scaffold, thereby optimizing human T cell activation [21]. 


\section{Structural Analysis of hPiezo1 Mutations}

\subsection{Sequence Alignment between hPiezo1 and mPiezo1}

As demonstrated in Figure 2, one of the three sequences that form the trimeric conformation of mPiezol can be roughly divided into domains termed as "Cap," $\mathrm{IH}, \mathrm{OH}$, Anchor, CTD, Latch, Beam, and Blade. The structure of Blade domain, due to the limitation of putative researches, is by far incomplete, providing only three Piezo repeats available.

One of the most prominent structures of Piezol should be the "Cap" domain. This portion of the molecule extends itself outside the cell membrane, which is a feature that endows the ability for it to sense a variety of mechanical movements outside of a cell [8]. With this specific location and connection with the pore inside, "Cap" is uniquely in charge of sensing shear flow, or shear stress, enabling the protein to carry out homeostatic processes in the blood [21] [28]. Besides, for "Cap" to be positioned outside of the cytosol and relatively mobile than most other domains, it has also been tested to participate of the "gate spring" activating mechanism: that is, a mechanism that can be mostly ascribed to contacts with the cytoskeleton [44] [45] [46]. What has also been linked to the "gating spring" mechanism is the Carbon-terminal Domain (CTD), and the Beam portion that extends from the Latch to the third Piezo Repeat. These domains are located inside the cytoplasm, which expediates their connections with the gating structures inside.

The Inner Helix $(\mathrm{IH})$ and the Outer Helix $(\mathrm{OH})$, primarily performs the mechanism of "annular lipids and agonists" along with Anchor-the neighboring domain-due to their locations around the entrances of the side channels of Piezo1. With annular or agonistic substances inside these channels, the hydrophobicity of the molecule changes and thus distorting the gated system, which could further lead to the opening of the gates [14] [15].

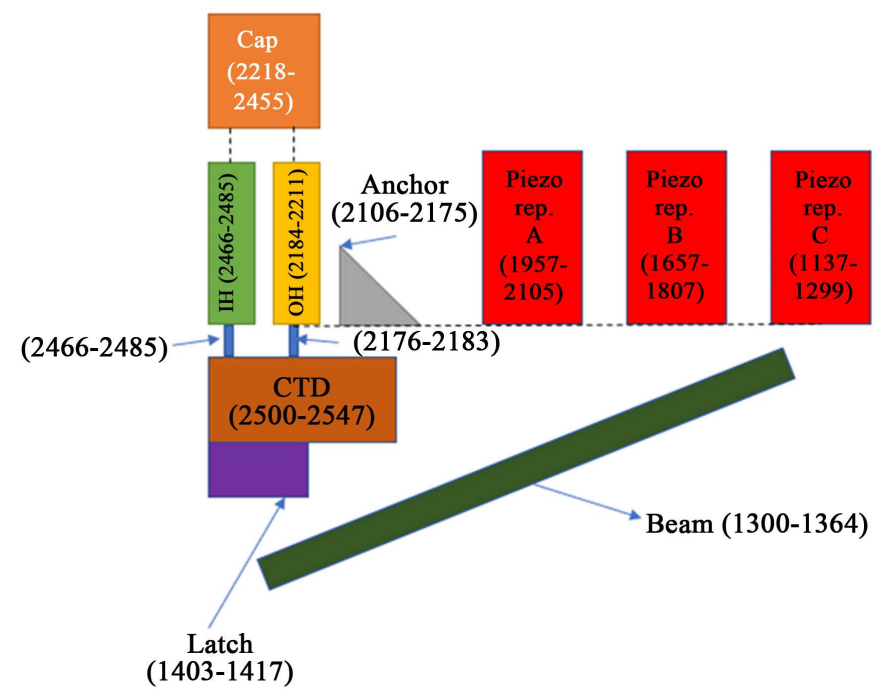

Figure 2. Schematic model of one sequence of mPiezo1 with domains categorized by residual codes. 
The effects of the membrane on a Piezol channel, on the other hand, are mostly the contribution from the Piezo Repeats inside, though some parts inside and outside, the lipid bilayer [8]. The functions of the membrane can be said as quite "versatile" since both thinning and curving can lead it to influence the gating of Piezo1 [15] [47] [48] [49] [50]. However, because of the incomplete information given on these Piezo repeats, their functions and relations to the gating system need further exploration.

As explicated by Saotome et al., the cap domain of mPiezolcorresponds to 2218 - 2455, Anchor 2106 - 2175, the latch 1403 - 1417, and the beam 1300 1364. Besides, Piezo1 repeats A, B, and C have been denoted as $1957-2105,1657$ - 1807, and 1137 - 1299 respectively. ThehumanPiezo1 (NCBIproteinID: 9606) has 2521 aminoacids, while the mouse Piezo1 (NCBI protein ID: 10090) has 2547 amino acids.hPiezo1 and mPiezo1 share over $80 \%$ sequence similarity as aligned by NCBI BLAST. To investigate the relationship between mutations and effects on structural parts, we match the reported human mutations [20] onto their coded positions on the mouse Piezo1 structure (Table 1).

Table 1. Mutation coded position and their correspondent domains and channel function.

\begin{tabular}{|c|c|c|}
\hline Position & $\begin{array}{l}\text { Correspondent } \\
\text { domain }\end{array}$ & Channel Function \\
\hline $2500-2547$ & CTD & Gating spring [3] [41] [42] [43] \\
\hline $2486-2499$ & CTD-IH Linkage & l \\
\hline $2466-2485$ & Inner Helix & Annular Lipids and Agonists [44] \\
\hline $2456-2465$ & Missing structure & l \\
\hline $2218-2455$ & “Cap" & Gating spring [3] [41] [42] [43] \\
\hline & & Shear flow sensing [18] [25] \\
\hline $2211-2217$ & Missing Structure & 1 \\
\hline $2184-2211$ & Outer Helix & Annular Lipids and Agonists [44] \\
\hline $2176-2183$ & CTD-OH Linkage & l \\
\hline $2106-2175$ & Anchor & Annular lipids and agonists [44] \\
\hline $1957-2105$ & Piezo Repeat A & $\begin{array}{l}\text { Hydrophobic mismatch by membrane curvature; } \\
\text { Hydrophobic mismatch by } \\
\text { membrane thinning [45] [46] [47] [48] [49] }\end{array}$ \\
\hline $1808-1956$ & Missing Structure & 1 \\
\hline $1657-1807$ & Piezo Repeat B & $\begin{array}{l}\text { Hydrophobic mismatch by membrane curvature; } \\
\text { Hydrophobic mismatch by } \\
\text { membrane thinning [45] [46] [47] [48] [49] }\end{array}$ \\
\hline $1418-1656$ & Missing Structure & 1 \\
\hline $1403-1417$ & Latch & l \\
\hline $1365-1402$ & Missing Structure & l \\
\hline $1300-1364$ & Beam & Gating spring [3] [41] [42] [43] \\
\hline $1137-1299$ & Piezo Repeat C & $\begin{array}{l}\text { Hydrophobic mismatch by membrane curvature; } \\
\text { Hydrophobic mismatch by } \\
\text { membrane thinning [45] [46] [47] [48] [49] }\end{array}$ \\
\hline
\end{tabular}


Over 25 mutations in hPiezo1 have been linked to multiple human disorders [19], while most mutations have not been characterized in detail. Having undergone the process of alignment using NCBI BLAST, the sequences and corresponding positions of mutated known amino acid residues of hPiezol have been mapped onto mPiezo1 (Table 2). The exception there is E1630X, which turns out to be D1621X if mapped on the mPiezo1 sequence. Thus, the study on hPiezo1 regarding this mutation would not be carried out here.

\subsection{UCSF Chimera Analysis of the hPiezo1 Mutation Sites Using mPiezo1 Template}

Since only the mPiezol protein was depicted in UCSF Chimera, we used mPiezo1 as template to analyze the effect of known mutations in hPiezolon the structural change of the whole protein. Due to the limitation on the decoded structure of mPiezo1, the mutations analyzed here will be those from S1153Trpfs * 21 downwards in Table 2. Moreover, S1153Trpfs * 21, K2166-2169 del, and E2496ELE are not workable in UCSF Chimera, so they are also suspended in this study. Besides, Y1763X, Q2244X, and P2456L temporarily lack corresponding structure in the model, which means this study is unable to edit their structures. Even though there are still a great many mutations worth analyzing, which are in italic in Table 2, with a totality of 14 of them.

The 14 mutations were applied to UCSF Chimera. Under the substitutions of rotamers, the mutations are simulated with mean angles and several possibilities recorded referencing Dunbrack 2010 library. Subsequently, contacts and clashes between the mutated residues and neighboring amino acid sequences are documented and then processed into Table 3. It has been tested that all identified residues have no clash or contact before mutation. The possibility of the contacts has also been quantified and with the number $>0.1$ been shown. Each mutation and its related clashes and contacts are discussed below:

Table 2. Correspondent mutations in mouse Piezo1 with human Piezo1.

\begin{tabular}{cccccc}
\hline $\begin{array}{c}\text { hPiezo1 } \\
\text { Mutation }\end{array}$ & $\begin{array}{c}\text { mPiezo1 } \\
\text { Mutation }\end{array}$ & $\begin{array}{c}\text { hPiezo1 } \\
\text { Mutation }\end{array}$ & $\begin{array}{c}\text { mPiezo1 } \\
\text { Mutation }\end{array}$ & $\begin{array}{c}\text { hPiezol } \\
\text { Mutation }\end{array}$ & $\begin{array}{c}\text { mPiezo1 } \\
\text { Mutation }\end{array}$ \\
\hline H702Y & H708Y & E1630X & D1621X & $V 2171 F$ & $V 2187 F$ \\
G718S & G724S & $V 1712 M$ & $V 1712 M$ & $M 2225 R$ & $M 2241 R$ \\
E755X & E749X & Y1763X & Y1763X & Q2228X & Q2244X \\
G782S & G777S & $R 1955 C$ & $R 1971 C$ & P2430L & $P 2456 L$ \\
R808Q & R803Q & $A 2003 D$ & $A 2019 D$ & $R 2456 H$ & $R 2482 H$ \\
L939M & L934M & $A 2020 T$ & $A 2036 T$ & $R 2456 C$ & $R 2482 C$ \\
I1007M & I1002M & $A 2020 V$ & $A 2036 V$ & $F 2458 L$ & $F 2484 L$ \\
S1117L & S1112L & $G 2029 R$ & $G 2045 R$ & $R 2488 Q$ & $R 2514 Q$ \\
S1153Trpfs $* 21$ & S1148Trpfs $* 21$ & $T 2127 M$ & $T 2143 \mathrm{M}$ & E2496ELE & E2522ELE \\
$R 1358 P$ & $R 1353 P$ & K2166-2169 del & K2182-2185 del & & \\
\hline
\end{tabular}


Table 3. Major residual contacts by mutation and respective possibilities.

\begin{tabular}{|c|c|c|}
\hline Mutation Code & Name of Contacted Residue & Possibility (\%) \\
\hline \multirow{2}{*}{$\mathrm{mR} 1353 \mathrm{P}$} & MET 1349 & 100.00 \\
\hline & LYS 1350 & 100.00 \\
\hline \multirow{2}{*}{$\mathrm{mV} 1712 \mathrm{M}$} & VAL 2054 & 30.97 \\
\hline & LEU 1716 & 22.04 \\
\hline $\mathrm{mR} 1971 \mathrm{C}$ & HIS 1334 & 100.00 \\
\hline $\mathrm{mA2019D}$ & TRP 2060 & 11.21 \\
\hline $\mathrm{mA} 2036 \mathrm{~T}$ & TRP 2142 & 100.00 \\
\hline \multirow{2}{*}{$\mathrm{mA} 2036 \mathrm{~V}$} & TRP 2142 & 100.00 \\
\hline & ILE 2033 & 55.32 \\
\hline \multirow{2}{*}{ mG2045R } & LEU 2037 & 100.00 \\
\hline & THR 2042 & 29.50 \\
\hline \multirow{4}{*}{$\mathrm{mT} 2143 \mathrm{M}$} & TRP 2152 & 39.52 \\
\hline & ASP 2139 & 37.49 \\
\hline & LEU 2106 & 22.92 \\
\hline & ARG 2514 & 12.11 \\
\hline \multirow[t]{2}{*}{$\mathrm{mV} 2187 \mathrm{~F}$} & ILE 2186 & 24.80 \\
\hline & GLU 2287 & 59.26 \\
\hline \multirow[t]{2}{*}{$\mathrm{mM} 2241 \mathrm{R}$} & ILE 2286 & 35.86 \\
\hline & SER 2289 & 21.17 \\
\hline $\mathrm{mR} 2482 \mathrm{H}$ & NONE & \\
\hline \multirow[t]{2}{*}{$\mathrm{mF} 2484 \mathrm{~L}$} & PHE 2485 & 10.90 \\
\hline & GLU 2522 & 24.57 \\
\hline \multirow[t]{2}{*}{$\mathrm{mR} 2514 \mathrm{Q}$} & TRP 2152 & 16.00 \\
\hline & PHE 2511 & 12.29 \\
\hline
\end{tabular}

\subsection{1. mR1353P (hR1358P) Mutation}

According to Table 3, after arginine at the position of 1353 mutates into proline, it forms contacts with two other residues, MET 1349 and LYS 1350, each at the possibility of $100 \%$ in sum. There exist two cases the Chi1, Chi2, and the possibility of each being $-27.2,37.7,0.813568$ and $19.6,-31.3,0.186432$, with the reference of Dunbrack 2010 library. The interactions between MET 1349 and PRO 1353 results from the contacts between $\mathrm{O}$ and $\mathrm{C}$ domains of the former and $\mathrm{CD}$ and CG domains of the latter. As seen from the data collected, the contacts between MET 1349 and PRO 1353, three in each of the two cases, have an average distance of about $2.14 \AA$.

The interactions between MET 1349 and LYS 1350, on the other hand, constitute of 6 inter-residual contacts with only one contact in the most probable $81.3568 \%$ case and 5 in another. The average distance between LYS 1350 and Proline 1353 stands at $2.84 \AA$ approximately. 


\subsection{2. mV1712M (hV1712M) Mutation}

The case of V1712M, with no Chi angle values in common amongst all the possibilities, is much more complicated. We found that there are 27 cases theoretically regarding this mutation, but one case with a possibility of 0 is excluded. Seven neighboring residues-one histidine, three leucine, and three valine-have been tested to be responsible for the contacts.

LEU 1716, appearing in four cases at a total probability of some $22 \%$, contacts CE and SD domains of MET 1712 with its CD1 and CD2 domains at an average distance of less than $2.5 \AA$, forming relatively stable interactions. The Chil value of rotamers that lead to the formation of contacts with LEU 1716 ranges from -178.4 to -134.1 , which is quite a restricted range. On the other hand, the Chi3 values that correlate with this bond formation are quite significant in magnitude $-108.8,170.9,-101$, and -173.6 .

LEU 1708, with the Chil value of MET 1712 increased, appear to be contacted. LEU 1716 has a variety of domains that participate in contact with the new rotamers, including $\mathrm{O}, \mathrm{C}, \mathrm{CB}$, and $\mathrm{CD} 2$. The average distance of bonds with LEU 1708 is about $2.23 \AA$, statistically averaged from 13 bonds in 4 rotamer cases whose Chi1 values range from -78.6 to 70.4 , in which there are also cases of "No Contacts."

Contacts with LEU 1713 manifest themselves when Chi1 values are relatively positive, from 65.3 to 76.3 , with three cases demonstrated to include these contacts, averaging a distance of $2.68 \AA$ by the total possibility of only 0.000014 . LEU 1713 embraces a great many domains that carry out these contacts: CG, $\mathrm{CD} 2, \mathrm{CD} 1, \mathrm{CB}, \mathrm{CA}$, and $\mathrm{N}$.

For Valine, VAL 2054 leads the list with a total emergence possibility of above 30\%. Like LEU 1716, VAL 2054 also happens to produce contacts when the Chi1 value of MET 1712 is quite negative at $-176.1,-171.0$, and -134.1 . The mean distance of the bonds between VAL 2054 and MET 1712 is at around $2.1645 \AA$ calculated from all eight contacts between CG1 and CB domains of VAL 2054 and CE and SD domains of MET 1712 rotamers.VAL 2055, immediately following with a $9.2479 \%$ possibility, occurs only in one case which has $-176.1,66.6$, and 74.7 as the three Chi values, making contacts together with VAL 2054 but at a further distance of $3.089 \AA$.

In contrast with the former two Valine residues, VAL 1709 is contacted when the Chil values of MET 1712 rotamers are comparatively positive, though contacts with VAL 1709 do form even when the rotamer has a Chil value of -72.4 with a chance of $0.1554 \%$. Under other circumstances, which sum up to be a mere $0.1291 \%$, Val 1709 makes contacts with the Chi1 value of MET 1712 rotamers at $68.9,70.4$, and 76.3 respectively, between which the average distance-standing at just over $2.1 \AA$-is relatively short.

Finally, HIS 2058 is only contacted for a probability of 0.000287 , forming four bonds with MET 1712 with a Chi1 value of -78.6.

Besides, there exist a total number of 14 "No Contacts" cases, which sum up to suggest a considerable proportion that may impede the mutations from affecting 
the structure of the protein. Therefore, further studies are required to match specific rotamers with their effects on the protein.

\subsection{3. mR1971C (R1955C) Mutation}

The mutation coded as R1971C is relatively straightforward. Only one residue, HIS 1334, is shown to be contacted after the mutation of ARG 1971 into Cysteine. On top of that, the contact between CYS 1971 and HIS 1334 happens in all cases, indicating a $100 \%$ probability of formation after this mutation.

\subsection{4. mA2019D (hA2003D) Mutation}

The mutation of A2019D seems to be as simple as the one of R1971C. However, though there is only one residue related in terms of contact formation, it only occurs by a possibility of about $11.2 \%$. The only residue involved, TRP 2060, interacts with ASP 2019 by its CZ2 and $\mathrm{CH} 2$ domains at an average distance of about $2.35 \AA$. Additionally, this contact is formed only when the Chil value of the Aspartic acid is -171.5 .

\subsection{5. mA2036T (hA2020T) Mutation}

The effects of A2036T mutation can mainly be attributed to the contacts with two amino acids-TRP 2142 and ILE 2033. TRP 2142 always contacts with THR 2036 independent of the case, whereas ILE 2033 produces interactions only at a possibility lower than $40 \%$. Also, as ILE 2033 forms only one contact in only one case, this study speculates that the major contributor of the structural and thus functional distortion to be TRP 2142.

\subsection{6. mA2036V (hA2020V) Mutation}

The Valine mutated from ALA 2036 creates contacts with precisely the same residues as the Tryptophan rotamers. Similarly, VAL 2036 makes bonds with TRP 2142 by a possibility of $100 \%$ and with ILE 2033 with a relatively low percentage of approximately $55.3 \%$. Though only two residues are involved in the formation of contacts after this mutation, TRP 2142 creates contacts with VAL 2036 by an extraordinarily high frequency of 14 contacts in all three cases, which illustrates its significance.

\subsection{7. mG2045R (hG2029R) Mutation}

The residues about the mutation of G2045R include nine residues in total. However, five of the residues are contacted by mere possibilities of far lower than 1\%. The residue that consistently makes contacts with ARG 2045 for this mutation is LEU 2037. Three cases with $0 \%$ possibilities exist for this mutation and are excluded from this discussion. Independent of the cases, an LEU 2037 CD1-ARG 2045 CB contact consistently forms by a uniform distance of $2.863 \AA$. Other contacted residues, on the other hand, form in specific Chi1 ranges. For example, THR 2042 is contacted had the Chil value of ARG 2045 been in the range of 54.1 to 85.3 with a total possibility of almost $30 \%$. Additionally, ARG 2040 is contacted by a non-negligible probability of about $8.73 \%$. To analyze the structural effects of this mutation, one needs to consider all these residues ac- 
cording to various cases.

\subsection{8. mT2143M (hT2127M) Mutation}

The mutation of $\mathrm{T} 2143 \mathrm{M}$ impacts a totality of eleven residues, including the one within a different sequence. The ones emerge by the prominent probabilities-all of them over 20\%-are ASP 2139, TRP 2152, and LEU 2106. Other residues, except ARG 2514, are of quite slight possibilities to be contacted-with not a single one exceeding $2 \%$. Interestingly, this mutation makes interactions with the residue from another chain, though only with a possibility of $0.0020 \%$; for example, the T2143M mutation within sequence A holds a possibility to contact with LYS 2188 within sequence C.

\subsection{9. mV2187F (hV2171F) Mutation}

The possibility for V2187F to yield contacts with other residues is relatively low, but the primary contact in this case-ILE 2186-still has a possibility of around $25 \%$ to be present. LYS 2183 and LYS 2188 have an identical possibility of $6.044 \%$ to emerging, whereas LYS 2184 slightly lower. V2187F contacts MET 2191 by a probability of approximately $8.5 \%$.

\subsubsection{0. mM2241R (hM2225R) Mutation}

Though contacting as many as 11 residues after the mutation, ARG 2241 mainly interacts with three residues-GLU 2287, ILE 2286, and SER 2289-by probabilities of about $60 \%, 35 \%$, and $20 \%$ respectively in a descending way. Other contacts with residues, led by the $7.4519 \%$ GLY 2288, seem to be dwarf under comparisons with the three protruded residues.

\subsubsection{1. mR2482H (hR2456H) Mutation}

Having undergone the mutation of R2482H, HIS 2482 forms contacts with neighboring residues with a comparatively low probability, with the highest among them being $21.1865 \%$ with GLY 2478 and SER 2486 following with a possibility of approximately $18.8 \%$. Also, the $14.6329 \%$ LYS 2479 cannot be said as insignificant for this mutation.

\subsubsection{2. mF2484L (hF2458L) Mutation}

Given that the probability for F2494L to contact VAL 2484 is just around 1\%, the sole effective contacted residue concerning this mutation is PHE 2485, which could be contacted with a possibility of approximately $10.9 \%$.

\subsubsection{3. mR2482C (hR2456C) Mutation}

No contacts have been formed after this mutation due probably to two reasons: 1) The default setting in this study does not cover the range wherein the contacts can be detected; 2) This mutation does not express itself in the structure but other alternative ways. Further studies need to be carried out to explicitly explain the relationship between this mutation and its corresponding disease.

\subsubsection{4. mR2514Q (hR2488Q) Mutation}

With ten residues possibly contacted in total and none of them exceeding a 25\% 
probability, the structural effect of this mutation should be described a complex and sporadic. The residue contacted with the highest probability-slightly lower than 25\%-is GLU 2522, TRP 2152 and PHE 2511 are the other two residues that are interacted by possibilities higher than $10 \%$, far greater than the other ones.

\section{Structure-to-Function Relationship between Mutations and Human Diseases}

\subsection{Structural Domains of Mutations and Contacts}

After being categorized and displayed, the positions of mutants and contacted structural parts can be analyzed as follows. In sum, four mutations happen within Piezo Repeat A, three within Inner Helix, two within Anchor, and 1 in each of Beam, Piezo Repeat B, Outer Helix, "Cap," and CTD.

For mutations within Piezo Repeat A, three of them form contacts within the domain itself. R1971C, as the only exception, only forms contacts with Beam, and the case of A2036V additionally makes connections with Anchor. The diseases related to mutations happening within Piezo Repeat A are versatile, with two cases pertaining to DHS and the other two to CAP and GLD respectively.

Mutations of Inner Helix, with a number of three, only have two of them producing identifiable contacts, both of which involving other residues within Inner Helix. Moreover, R2482H yield interactions with Anchor and CTD-IH Linkage as well. Interestingly, all three of these Inner-Helical mutations-by prediction-will lead to GLD. Nevertheless, the structural impact of R2482C has not been identified, a lack of which indicates further research.

Within Anchor, two mutations trigger contacts with three domains: Piezo Repeat A, Anchor, and CTD. Both cases of Anchor mutations are estimated to give rise to DHS.

The only mutations happening within each of the other domains-including CTD, "Cap," Beam, and Piezo Repeat B, and Outer Helix-have all been demonstrated to contact with residues from these domains. CTD and Piezo Repeat B, however, additionally form contacts with Anchor and Piezo Repeat A, respectively.

\subsection{Relationship between Mutations and Human Diseases}

Given all the information described above, it is possible for us to make links between structural contacts from mutations and the formation of diseases. To make the analysis ordered and bright, this study will discuss the aforementioned mutations in the categories of three diseases.

First, dehydrated hereditary stomatocytosis (DHS) damages the function of human RBCs by making it abnormally permeable to monovalent cations and thus triggering osmotic responses [51]. For mutations related to DHS, both the Piezo Repeats (mainly A and B) and the Anchor domain play crucial roles. Among all seven mutations that could lead to DHS, four include the interaction between the anchor and Piezo Repeat A, which is possibly a significant cause of DHS. The mutations in the Piezo Repeats would most likely affect the activation 
mechanisms related to the plasma membrane, with information in Table 1 as reference. The distortion in the Beam domain can lead to abnormal curvature and thickness of the Piezol membrane, thus increasing the extent of effect for channel activation to occur, as suggested by the "Gravitation Model" [9]. By this means, cations are less likely to flow inside the cell, a phenomenon that leads to the destruction of the osmotic balance of erythrocytes so that water flows outside the cytoplasm and the cells shrink. On the other hand, the Anchor domain of Piezo1, if having a part of it mutated, would alter the structure of the central pore, influencing the central pore and hence ion movements. In the case of R2488Q, a mutation in CTD that can lead to DHS, the contacted structural portion is the Anchor.

Second, colorectal adenomatous polyposis (CAP) is closely related to Hamartomatous polyps covered by a layer of the hypertrophic epithelium [52]. These epithelium cells, usually beneath secreted mucus, could be affected by mutations in Piezo1 molecules. To be hypertrophic, as previously stated as part of the CAP syndrome, these epithelium cells should have an abnormally high concentration of ions inside them, which is believed to be done by a loosening in the Piezol gating. According to Table 4, the formation of CAP is primarily related to

Table 4. Structural domains of mutations and contacts, and diseases related by prediction.

\begin{tabular}{|c|c|c|c|}
\hline Structural domains of mutation & Mutation Code & Contacted Domains & Diseases Related \\
\hline \multirow{5}{*}{ Piezo Repeat A } & hR1955C & Beam & CAP \\
\hline & hA2020T & Piezo Repeat A & DHS \\
\hline & \multirow{2}{*}{ hA2020V } & Anchor & \multirow{2}{*}{ DHS } \\
\hline & & Piezo Repeat A & \\
\hline & hG2029R & Piezo Repeat A & GLD \\
\hline \multirow{5}{*}{ Inner Helix } & \multicolumn{3}{|c|}{ Inner Helix } \\
\hline & $\mathrm{hR} 2482 \mathrm{H}$ & Anchor & GLD \\
\hline & \multicolumn{3}{|c|}{ CTD-IH Linkage } \\
\hline & hR2456C & None & GLD \\
\hline & hF2458L & Inner Helix & GLD \\
\hline \multirow{3}{*}{ Anchor } & hA2003D & Piezo Repeat A & DHS \\
\hline & \multirow{2}{*}{ hT2127M } & Anchor & \multirow{2}{*}{ DHS } \\
\hline & & CTD & \\
\hline \multirow{2}{*}{ CTD } & \multirow{2}{*}{ hR2488Q } & CTD & \multirow{2}{*}{ DHS } \\
\hline & & Anchor & \\
\hline “Cap" & hM2225R & “Cap" & DHS \\
\hline Beam & hR1358P & Beam & DHS \\
\hline \multirow{2}{*}{ Piezo Repeat B } & \multirow{2}{*}{ hV1712M } & Piezo Repeat A & \multirow[b]{2}{*}{ CAP } \\
\hline & & Piezo Repeat B & \\
\hline Outer Helix & hV2171F & Outer Helix & GLD \\
\hline
\end{tabular}


contacts within the Blade domain and between Blade and Beam. That is, the excessive entering of ions into the cell relates to the alteration of the functions related to the plasma membrane, as seen from the correlation demonstrated in Table 3. What is as well worth mentioning is the mechanisms pertained to the Beam domain, though the sample size may be overly small for thorough analysis. It can be logically proposed that cytoskeleton and the so-called "Gating Spring" mechanism may also contribute to the broader opening of the Piezol gating system, thus allowing in a higher number of ions and triggering the osmotic imbalance. With only two mutations related to CAP disease, both V1712M and R1955C seem to play a role in the structural distortion of the Piezo Repeats, indicating the effect on the Beam, arisen from the R1955C mutation, should not be overlooked.

In addition, generalized lymphatic dysplasia (GLD), the dysfunction of a part of the lymphatic system, leads to symptoms such as "intestinal and/or pulmonary lymphangiectasia, pleural effusions, chylothoraces and/or pericardial effusions" as suggested by Foutiou et al. [28]. This leakage of fluid from the inside of the cells is also of homeostatic importance. The outward flow of the liquid can arguably be ascribed to the increase in the water potential of the cytosol. Displayed in Table 4, GLD seems to correlate strongly with the central bundles of $\mathrm{IH}$ and $\mathrm{OH}$. The alteration in these two structures reasonably affects, probably clutches, the central pore of Piezo1, therefore letting in ions that are of too little an amount. That said, the component of Piezo Repeat A cannot be overlooked, as it may also relate to the operation of the central pore of the molecule.

\section{Conclusions}

This study has explored the structural impacts of residual mutations within the Piezo1 molecule, with the mutations, as well as their related diseases. NCBI BLAST has aligned Piezo1 sequences between humans and mice, empowering this study to analyze structural changes in the Piezol protein through UCSF Chimera.

With the aid from UCSF Chimera, this study has recorded and interpreted clashes and contacts originated from each of the mutations. The discussion of the results about structural changes has included distances between contacted residues, names of contacted residues, and the possibilities for these residues to be involved in the contacts. After the discussion on primary data collected, the linkages between Piezol's structural parts have been investigated as residues-mutated or contacted-are categorized by the sequence ranges of various portions of the protein. Given these discussions and classifications, this research has indicated the most prominent structural connections that could probably lead to the formation of diseases, including DHS, CAP, and GLD.

With all these investigations done above, the connections between the structure of Piezol and three human diseases correlated have been roughly set up. These connections, though still requiring further experimentation to corroborate, propose the major ways in which alterations in the structure of Piezol af- 
fect its operation and point out a further direction of related studies in the future, which could hopefully go beyond the limit of technical and theoretical restrictions.

\section{Acknowledgements}

This research was supported by GEC Academy.

\section{Conflicts of Interest}

The author declares that he has no competing interests.

\section{References}

[1] Xiao, R. and Xu, X.S. (2010) Mechanosensitive Channels: In Touch with Piezo. Current Biology, 20, R936-R938. https://doi.org/10.1016/j.cub.2010.09.053

[2] Coste, B., et al. (2010) Piezo1 and Piezo2 Are Essential Components of Distinct mechanically Activated Cation Channels. Science, 330, 55-60. https://doi.org/10.1126/science.1193270

[3] Coste, B., et al. (2012) Piezo Proteins Are Pore-Forming Subunits of Mechanically Activated Channels. Nature, 483, 176. https://doi.org/10.1038/nature10812

[4] Alper, S. (2017) Genetic Diseases of PIEZO1 and PIEZO2 Dysfunction. In: Current Topics in Membranes, Elsevier, Amsterdam, 97-134.

https://doi.org/10.1016/bs.ctm.2017.01.001

[5] Parpaite, T. and Coste, B. (2017) Piezo Channels. Current Biology, 27, R250-R252. https://doi.org/10.1016/j.cub.2017.01.048

[6] Moroni, M., et al. (2018) Voltage Gating of Mechanosensitive PIEZO Channels. Nature Communications, 9, Article No. 1096. https://doi.org/10.1038/s41467-018-03502-7

[7] Volkers, L., Mechioukhi, Y. and Coste, B. (2015) Piezo Channels: From Structure to Function. Pflügers Archiv. European Journal of Physiology, 467, 95-99. https://doi.org/10.1007/s00424-014-1578-Z

[8] Ge, J., et al. (2015) Architecture of the Mammalian Mechanosensitive Piezol Channel. Nature, 527, 64-69. https://doi.org/10.1038/nature15247

[9] Guo, Y.R. and MacKinnon, R. (2017) Structure-Based Membrane Dome Mechanism for Piezo Mechanosensitivity. Elife, 6, e33660. https://doi.org/10.7554/eLife.33660

[10] Saotome, K., et al. (2018) Structure of the Mechanically Activated Ion Channel Piezo1. Nature, 554, 481. https://doi.org/10.1038/nature25453

[11] Zhao, Q., et al. (2018) Structure and Mechanogating Mechanism of the Piezo1 Channel. Nature, 554, 487-492. https://doi.org/10.1038/nature25743

[12] Zhao, Q., et al. (2018) The Mechanosensitive Piezo1 Channel: A Three-Bladed Propeller-Like Structure and a Lever-Like Mechanogating Mechanism. The FEBS Journal, 286, 2461-2470. https://doi.org/10.1111/febs.14711

[13] Wang, Y., et al. (2018) A Lever-Like Transduction Pathway for Long-Distance Chemical- and Mechano-Gating of the Mechanosensitive Piezol Channel. Nature Communications, 9, Article No. 1300. https://doi.org/10.1038/s41467-018-03570-9

[14] Jia, Z., et al. (2016) Regulation of Piezo2 Mechanotransduction by Static Plasma Membrane Tension in Primary Afferent Neurons. Journal of Biological Chemistry, 
291, 9087-9104. https://doi.org/10.1074/jbc.M115.692384

[15] Borbiro, I., Badheka, D. and Rohacs, T. (2015) Activation of TRPV1 Channels Inhibits Mechanosensitive Piezo Channel Activity by Depleting Membrane Phosphoinositides. Science Signaling, 8, ra15. https://doi.org/10.1126/scisignal.2005667

[16] Bavi, O., et al. (2016) Influence of Global and Local Membrane Curvature on Mechanosensitive Ion Channels: A Finite Element Approach. Membranes, 6, 14. https://doi.org/10.3390/membranes6010014

[17] Wang, Y. and Xiao, B. (2018) The Mechanosensitive Piezol Channel: Structural Features and Molecular Bases Underlying Its Ion Permeation and Mechanotransduction. The Journal of Physiology, 596, 969-978. https://doi.org/10.1113/JP274404

[18] French, A. (1992) Mechanotransduction. Annual Review of Physiology, 54, 135-152. https://doi.org/10.1146/annurev.ph.54.030192.001031

[19] Wu, J., Lewis, A.H. and Grandl, J. (2017) Touch, Tension, and Transduction: The Function and Regulation of Piezo Ion Channels. Trends in Biochemical Sciences, 42, 57-71. https://doi.org/10.1016/j.tibs.2016.09.004

[20] Pathak, M.M., et al. (2014) Stretch-Activated Ion Channel Piezo1 Directs Lineage Choice in Human Neural Stem Cells. Proceedings of the National Academy of Sciences, 111, 16148-16153. https://doi.org/10.1073/pnas.1409802111

[21] Cinar, E., et al. (2015) Piezo1 Regulates Mechanotransductive Release of ATP from Human RBCs. Proceedings of the National Academy of Sciences, 112, 11783-11788. https://doi.org/10.1073/pnas.1507309112

[22] Li, C., et al. (2015) Piezo1 Forms Mechanosensitive Ion Channels in the Human MCF-7 Breast Cancer Cell Line. Scientific Reports, 5, Article No. 8364. https://doi.org/10.1038/srep08364

[23] Jin, Y., et al. (2014) Functional Role of Mechanosensitive Ion Channel Piezo1 in Human Periodontal Ligament Cells. The Angle Orthodontist, 85, 87-94. https://doi.org/10.2319/123113-955.1

[24] Liu, C.S.C., et al. (2018) Cutting Edge: Piezo1 Mechanosensors Optimize Human T Cell Activation. The Journal of Immunology, 200, 1255-1260. https://doi.org/10.4049/jimmunol.1701118

[25] Shmukler, B.E., et al. (2015) Homozygous Knockout of the piezol Gene in the Zebrafish Is Not Associated with Anemia. Haematologica, 100, e483. https://doi.org/10.3324/haematol.2015.132449

[26] Ranade, S.S., et al. (2014) Piezo1, a Mechanically Activated Ion Channel, Is Required for Vascular Development in Mice. Proceedings of the National Academy of Sciences, 111, 10347-10352. https://doi.org/10.1073/pnas.1409233111

[27] Martins, J.R., et al. (2016) Piezo1-Dependent Regulation of Urinary Osmolarity. Pflügers Archiv. European Journal of Physiology, 468, 1197-1206. https://doi.org/10.1007/s00424-016-1811-z

[28] Rode, B., et al. (2017) Piezol Channels Sense Whole Body Physical Activity to Reset Cardiovascular Homeostasis and Enhance Performance. Nature Communications, 8, Article No. 350.

[29] Faucherre, A., et al. (2014) Piezo1 Plays a Role in Erythrocyte Volume Homeostasis. Haematologica, 99, 70-75. https://doi.org/10.3324/haematol.2013.086090

[30] Ma, S., et al. (2018) Common PIEZO1 Allele in African Populations Causes RBC Dehydration and Attenuates Plasmodium Infection. Cell, 173, 443-455e12. https://doi.org/10.1016/j.cell.2018.02.047

[31] Fotiou, E., et al. (2015) Novel Mutations in PIEZO1 Cause an Autosomal Recessive 
Generalized Lymphatic Dysplasia with Non-Immune Hydrops Fetalis. Nature Communications, 6, Article No. 8085. https://doi.org/10.1038/ncomms 9085

[32] Nonomura, K., et al. (2018) Mechanically Activated Ion Channel PIEZO1 Is Required for Lymphatic Valve Formation. Proceedings of the National Academy of Sciences, 115, 12817-12822. https://doi.org/10.1073/pnas.1817070115

[33] Lukacs, V., et al. (2015) Impaired PIEZO1 Function in Patients with a Novel Autosomal Recessive Congenital Lymphatic Dysplasia. Nature Communications, 6, Article No. 8329. https://doi.org/10.1038/ncomms9329

[34] Lee, W., et al. (2014) Synergy between Piezo1 and Piezo2 Channels Confers High-Strain Mechanosensitivity to Articular Cartilage. Proceedings of the National Academy of Sciences, 111, E5114-E5122. https://doi.org/10.1073/pnas.1414298111

[35] Bae, C., et al. (2013) Xerocytosis Is Caused by Mutations That Alter the Kinetics of the Mechanosensitive Channel PIEZO1. Proceedings of the National Academy of Sciences, 110, E1162-E1168. https://doi.org/10.1073/pnas.1219777110

[36] Demolombe, S., et al. (2013) Slower Piezo1 Inactivation in Dehydrated Hereditary Stomatocytosis (Xerocytosis). Biophysical Journal, 105, 833.

https://doi.org/10.1016/j.bpj.2013.07.018

[37] Glogowska, E., et al. (2017) Novel Mechanisms of PIEZO1 Dysfunction in Hereditary Xerocytosis. Blood, 130, 1845-1856. https://doi.org/10.1182/blood-2017-05-786004

[38] Andolfo, I., et al. (2015) Novel Gardos Channel Mutations Linked to Dehydrated Hereditary Stomatocytosis (Xerocytosis). American Journal of Hematology, 90, 921-926. https://doi.org/10.1002/ajh.24117

[39] Zarychanski, R., et al. (2012) Mutations in the Mechanotransduction Protein PIEZO1 Are Associated with Hereditary Xerocytosis. Blood, 120, 1908-1915. https://doi.org/10.1182/blood-2012-04-422253

[40] Sandberg, M., et al. (2014) Hereditary Xerocytosis and Familial Haemolysis Due to Mutation in the PIEZO1 Gene: A Simple Diagnostic Approach. International Journal of Laboratory Hematology, 36, e62-e65. https://doi.org/10.1111/ijlh.12172

[41] Romac, J.M.-J., et al. (2018) Piezo1 Is a Mechanically Activated Ion Channel and Mediates Pressure Induced Pancreatitis. Nature Communications, 9, Article No. 1715. https://doi.org/10.1038/s41467-018-04194-9

[42] Sun, W., et al. (2019) The Mechanosensitive Piezol Channel Is Required for Bone Formation. eLife, 8, e47454. https://doi.org/10.1016/bs.ctm.2016.11.004

[43] Gnanasambandam, R., Gottlieb, P.A. and Sachs, F. (2017) The Kinetics and the Permeation Properties of Piezo Channels. Current Topics in Membranes, 79, 275-307.

[44] Wu, J., Goyal, R. and Grandl, J. (2016) Localized Force Application Reveals Mechanically Sensitive Domains of Piezo1. Nature Communications, 7, Article No. 12939. https://doi.org/10.1038/ncomms12939

[45] Cox, C.D., et al. (2016) Removal of the Mechanoprotective Influence of the Cytoskeleton Reveals PIEZO1 Is Gated by Bilayer Tension. Nature Communications, 7, Article No. 10366. https://doi.org/10.1038/ncomms10366

[46] Nourse, J.L. and Pathak, M.M. (2017) How Cells Channel Their Stress: Interplay between Piezol and the Cytoskeleton. Seminars in Cell \& Developmental Biology, 71, 3-12. https://doi.org/10.1016/j.semcdb.2017.06.018

[47] Janmey, P. and Kinnunen, P. (2006) Biophysical Properties of Lipids and Dynamic Membranes. Trends in Cell Biology, 16, 538-546. 
https://doi.org/10.1016/j.tcb.2006.08.009

[48] Lewis, A.H. and Grandl, J. (2015) Mechanical Sensitivity of Piezol Ion Channels Can Be Tuned by Cellular Membrane Tension. Elife, 4, e12088.

https://doi.org/10.7554/eLife.12088

[49] Pliotas, C., et al. (2015) The Role of Lipids in Mechanosensation. Nature Structural \& Molecular Biology, 22, 991. https://doi.org/10.1038/nsmb.3120

[50] Lolicato, M., et al. (2014) Transmembrane Helix Straightening and Buckling Underlies Activation of Mechanosensitive and Thermosensitive K2P Channels. Neuron, 84, 1198-1212. https://doi.org/10.1016/j.neuron.2014.11.017

[51] Delaunay, J. (2004) The Hereditary Stomatocytoses: Genetic Disorders of the Red Cell Membrane Permeability to Monovalent Cations. Seminars in Hematology, 41, 165-172. https://doi.org/10.1053/j.seminhematol.2004.02.005

[52] Shussman, N. and Wexner, S.D. (2014) Colorectal Polyps and Polyposis Syndromes. Gastroenterology Report, 2, 1-15. https://doi.org/10.1093/gastro/got041

\author{
Abbreviations \\ CAP: Colorectal adenomatous polyposis \\ CED: Carbon-terminal extracellular domain \\ CTD: Carbon-terminal domain \\ DHS: Dehydrated hereditary xerocytosis \\ EV: Extracellular vestibule \\ GLD: Generalized lymphatic dysplasia \\ IH: Inner Helix \\ IV: Intracellular vestibule \\ MV: Membrane vestibule \\ OH: Outer helix \\ RBC: Red blood cell \\ TM: Transmembrane \\ THU: Transmembrane helical units
}

